\title{
Downlink Performance Analysis of Distributed Antenna Systems with Linear Precoding
}

\author{
Chuanlei SONG ${ }^{1}$, Pengcheng $\mathrm{ZHU}^{1}$, Jiamin $\mathrm{LI}^{1}$ \\ ${ }^{1}$ Mobile Communications Research Laboratory, Southeast University,
}

Nanjing, 210096, China

email: p.zhu@seu.edu.cn

Keywords: DAS, linear precoding, optimal precoding

\begin{abstract}
Distributed antenna system (DAS) has emerged as a promising architecture for future mobile communications. Compared with collocated antenna system (CAS), DAS has advantages in reducing transmission power and improving the cell coverage area. In this paper, the downlink performance of DAS with linear precoding is analyzed. The optimization problem of minimizing the total transmitted power while satisfying signal-to-interference-and-noise ratio (SINR) constraints is studied to optimize the beamforming for all user jointly, which can be solved by conic optimization method. However, the problem is non-convex and can not be solved efficiently. Suboptimal methods are proposed by optimizing power allocation after given the direction of precoding. In simulation, the performance of different linear precoding methods are analyzed for the downlink of DAS. Moreover, the performance of DAS and CAS are also compared with the same antenna configuration and direction of precoding. It is shown that compared with CAS, DAS achieves the same performance with much less transmission power.
\end{abstract}

\section{Introduction}

More attention was paid to the distributed antenna system (DAS) because of its potential to improve system coverage and reduce the energy consumption. In DAS, the remote antenna units (RAUs) are separately and remotely located in a cell in accordance with certain rules. The RAUs are connected with the baseband processing unit (BPU) via Fiber-optic backbone network or dedicated radio link [1]. By introducing the RAUs, the distance between the mobile terminals (MTs) and the antennas of the RAU is greatly reduced. Thus, the large path loss of the radio signals can be avoided. Furthermore, the RAUs are located in different locations, which can be used to form a distributed multiple-input multiple-output (MIMO) system to further improve the performance of radio links.

DAS has been extensively studied since 1980s. [2] proposed the idea of distributed antenna system, for solving the "blind spot" and large-scale indoor wireless communications fading problem. [3] pointed out that the DAS can be used to improve the capacity and coverage of the cell. [4] analyzed the capacity of CDMA DAS. In [5], the authors compared the general distributed antenna system (GDAS) and conventional cellular system where the same conclusion as [3] was drawn.

In the downlink of DAS, the request for better performance with less complexity lead researchers to optimize the transmitter. Linear precoding, as the key technology to reduce the interference and maximize the throughput, has been extensively studied. Linear precoding mainly consists of maximum ratio transmission (MRT) precoding, zero forcing precoding (ZF) and signal-to-leakage-and-noise ratio (SLNR) precoding [6] [7] [8]. In order to improve the performance, many researchers proposed the optimal precoding according different criterions. [12] proposed a solution to solve the multiuser downlink problem with individual SINR constraints in a multiple-input single-output (MISO) system. The algorithm is based on the duality between the uplink and the downlink, and solved the problem iteratively in the uplink before switching to the downlink. [13] proposed a solution to solve the multiuser downlink problem with SINR constraints in a MIMO system. The algorithm is also based on the duality between the uplink and the downlink. For a change, [9] utilized convex optimization method to solve the multiuser downlink problem 
with the transmitted power constraints in MISO system.

In this paper, we introduce convex optimization method to solve the multiuser downlink problem with SINR constraints in DAS. In order to reduce complexity, suboptimal methods are proposed by optimizing power allocation after given the direction of precoding. The performance of DAS with optimal precoding method and suboptimal precoding methods are analyzed. In addition, the performance of DAS and CAS are also compared with the same antenna configuration and direction of precoding. Numerical results show that the performance of DAS is better that of CAS.

The following notations are used in this paper. Standard lower case letters (e.g. a) denotes scalars, boldface lower case letters (e.g. a) denotes column vectors and boldface upper case letters (e.g. A) denotes matrices. $\mathbf{I}_{M}$ denotes an identity matrix of size $M$ and $\operatorname{diag}($.) denotes diagonal matrix. $(.)^{\mathrm{T}},(.)^{\mathrm{H}}$ and $(.)^{\dagger}$ denote the transpose, the conjugate transpose and pseudo-inverse, respectively. $\mathrm{E}($.$) denotes the mean, \mathrm{N}\left(\mu, \sigma^{2}\right)$ and $\mathrm{CN}\left(\mu, \sigma^{2}\right)$ denote respectively Gaussian distribution and complex Gaussian distribution, which $\mu$ is mean and $\sigma^{2}$ is variance.

\section{System Model}

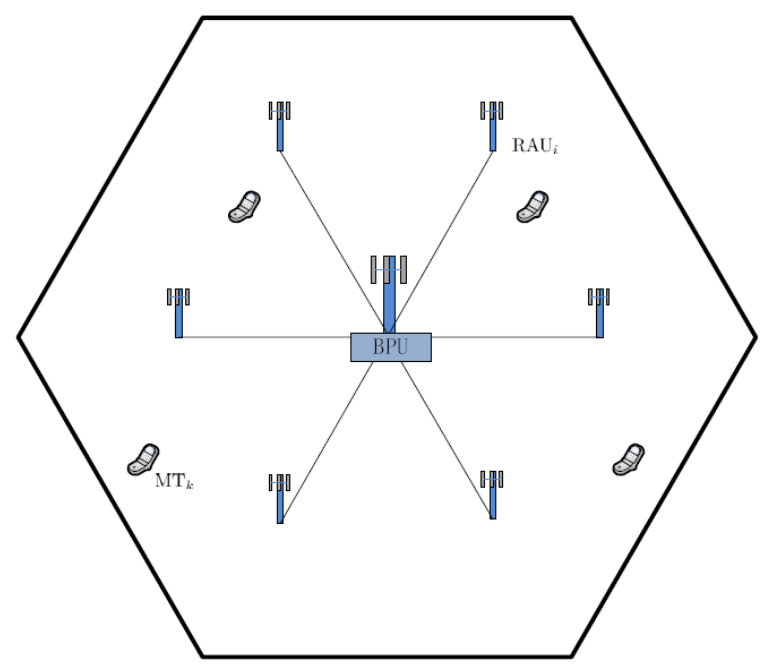

Fig. 1. A generalized distributed antenna system.

As shown in Fig. 1, a GDAS consisting of $N$ RAUs and each RAU is equipped with $L$ antennas is considered. We assumed that the radius of GDAS is $D \mathrm{Km}$ and the location of the RAU is fixed. There are K MTs randomly distributed in the cell and each MT uses a single antenna. In the downlink, $\mathbf{h}_{k, n}=\left[h_{k, n, 1} \ldots h_{k, n, L}\right]^{\mathrm{T}}$ denotes the channel gain from the $n$-th RAU to the $k$-th MT. $h_{k, n, l}$ is modeled as

$$
h_{k, n, l}=\sqrt{c d_{k, n, l}^{-\alpha} s_{k, n, l}} v_{k, n, l}
$$

where $c d_{k, n, l}^{-\alpha}$ denotes the pass loss, $c$ denotes the median of the mean path loss at the reference distance of $d_{0}, d_{k, n, l}$ denotes the distance between the $k$-th MT and the $l$-th antenna in the $n$-th RAU, $\alpha$ is the pass loss exponent. denotes the large scale fading and is a log-normal distributed random variable, i.e, $10 \log _{10}\left(s_{k, n, l}\right) \square N\left(0, \sigma^{2}\right), \sigma^{2}$ is the variance, $v_{k, n, l}$ denotes the small-scale fading and is a circularly symmetric complex Gaussian random variables with zero mean and unit variance, i.e, $v_{k, n, l} \square C N(0,1)$. In (1), the variables, $d_{k, n, l}, s_{k . n . l}$ and $v_{k, n, l}$ are assumed to be independent of each other. In the downlink, the received signal of the $k$-th MT is

$$
y_{k}=\mathbf{h}_{k}^{\mathrm{H}} \mathbf{x}+z_{k}
$$

where $\mathbf{h}_{k}=\left[\mathbf{h}_{k, 1} \mathbf{h}_{k, 2} \ldots \mathbf{h}_{k, N}\right]^{\mathrm{T}}$ denotes the channel gain vector from all RAUs to the $k$-th MT, $z_{k}$ is complex additive white Gaussian noise (AWGN) with zero mean and variance $\sigma^{2}$, i.e, $v_{k, n, l} \square C N(0,1) \cdot \mathbf{x}$ denoting the transmitted symbols, can be written as

$$
\mathbf{x}=\mathbf{T s}
$$


Where $\mathbf{T}=\left[\mathbf{t}_{1}, \ldots, \mathbf{t}_{K}\right]^{\mathrm{T}}$ denotes the precoding matrix. $\mathbf{s}=\left[s_{1}, \ldots, s_{K}\right]$ denotes transmitted information signal satisfying $\mathrm{E}\left(\mathbf{s s}^{\mathrm{H}}\right)=\mathbf{I}_{K}$. Then the overall downlink input-output relationship is given by

$\mathbf{y}=\mathbf{H T s}+\mathbf{z}$

where $\mathbf{y}=\left[y_{1}, \ldots, y_{K}\right]^{\mathrm{T}}, \mathbf{H}=\left[\mathbf{h}_{1}, \ldots, \mathbf{h}_{K}\right]^{\mathrm{T}}, \mathbf{z}=\left[\mathrm{z}_{1}, \ldots, \mathbf{z}_{K}\right]^{\mathrm{T}}$.

\section{Precoding Method}

According to the downlink channel information, the transmitter designs precoding matrix to eliminate interference between the MTs. The design of precoding is mostly based on minimizing mean square error (MMSE) criterion and maximizing SINR criterion. The MMSE criterion performs quite well but is usually computationally. When the interesting and relevant criteria are bit error rate (BER) and capacity that are intimately associated with maximizing SINR, the SINR criterion can be considered. The SINR criterion have two strategies: The first maximizes the SINR subject to an average power constraint, and the second minimizes the required average power subject to a constraint on the worst SINR. In this paper, the second strategies is adopted, which can be expressed as

$$
\begin{array}{lll} 
& \min _{\mathbf{t}_{1}, \ldots, \mathbf{t}_{K}} & \sum_{k=1}^{K}\left\|\mathbf{t}_{k}\right\|^{2} \\
\text { subject } & \text { to } & \text { SINR }_{k} \geq \gamma_{0}, k=1, \ldots, K
\end{array}
$$

Where $\gamma_{0}$ is SINR constraint. In this paper, the optimal precoding and the suboptimal precoding are designed according to (5).

\section{A. Optimal Precoding}

In this paper, convex optimization method is used for solving the problem of (5). First, the mathematical expression of SINR is

$$
\operatorname{SINR}_{k}=\frac{\left|\mathbf{h}_{k}^{\mathrm{H}} \mathbf{t}_{k}\right|^{2}}{\sum_{i \neq k}^{K}\left|\mathbf{h}_{k}^{\mathrm{H}} \mathbf{t}_{i}\right|^{2}+\sigma_{k}^{2}}
$$

According to (6), the problem (5) can be expressed as

$$
\min _{\mathbf{t}_{1}, \ldots, \mathbf{t}_{K}} \sum_{k=1}^{K}\left\|\mathbf{t}_{k}\right\|^{2}
$$

subject to $\frac{1}{\gamma_{0}}\left|\mathbf{h}_{k}^{\mathrm{H}} \mathbf{t}_{k}\right|^{2} \geq \sum_{i \neq k}\left|\mathbf{h}_{k}^{\mathrm{H}} \mathbf{t}_{i}\right|^{2}+\sigma_{k}^{2}, k=1, \ldots, K$

The problem (7) is not convex problem and the KKT conditions are not sufficient for optimality in non-convex programs. However, in Appendix A, it shows that in this special case, if the problem is strictly feasible, then its KKT conditions are necessary and sufficient for optimality. The Lagrangian associated with problem (7) is given by

$$
\mathrm{L}\left(\mathbf{t}_{1}, \ldots, \mathbf{t}_{K} ; \mu_{1}, \ldots, \mu_{K}\right)=\sum_{k=1}^{K}\left\|\mathbf{t}_{k}\right\|^{2}+\sum_{k=1}^{K} \mu_{k}\left(\sum_{i=1}\left|\mathbf{h}_{k}^{\mathrm{H}} \mathbf{t}_{i}\right|^{2}+\sigma_{k}^{2}-\left(1+\frac{1}{\gamma_{0}}\right)\left|\mathbf{h}_{k}^{\mathrm{H}} \mathbf{t}_{k}\right|^{2}\right)
$$

Where $\mu_{k} \geq 0, k=1, \ldots, K$ are the Lagrange dual variables. Here, the stationary KKT conditions that say that $\partial L / \partial \mathbf{t}_{k}=\mathbf{0}, k=1, \ldots, K$ as the optimal solution is exploited and we can get

$$
\mathbf{t}_{k}+\sum_{i \neq k} \frac{\mu_{i}}{\sigma_{k}^{2}} \mathbf{h}_{i} \mathbf{h}_{i}^{\mathrm{H}} \mathbf{t}_{k}-\frac{\mu_{k}}{\gamma_{0} \sigma_{k}^{2}} \mathbf{h}_{k} \mathbf{h}_{k}^{\mathrm{H}} \mathbf{t}_{k}=\mathbf{0}
$$

We define $p_{k}$ as the transmission power for $k$-th MT. Finally, the optimal precoding vector was:

$$
\mathbf{t}_{k}=\frac{\left(\mathbf{I}_{N}+\sum_{i=1}^{K} \frac{\mu_{i}}{\sigma_{k}^{2}} \mathbf{h}_{i} \mathbf{h}_{i}^{\mathrm{H}}\right)^{-1} \mathbf{h}_{k}}{\left\|\left(\mathbf{I}_{N}+\sum_{i=1}^{K} \frac{\mu_{i}}{\sigma_{k}^{2}} \mathbf{h}_{i} \mathbf{h}_{i}^{\mathrm{H}}\right)^{-1} \mathbf{h}_{k}\right\|} \sqrt{p_{k}}, k=1, \ldots, K
$$


The precoding design is based on the powerful framework of convex optimization theory, which allows for efficient numerical solutions using standard optimization packages. Specifically, the optimization problem can be formulated as a second-order cone program (SOCP) [10] or a semi-definite program (SDP) [11].

\section{B. Suboptimal Precoding}

From (10), the precoding vector includes direction vector and power. It is foreseeable that the complexity of optimal precoding will decrease when the direction of precoding vector is sure. Suboptimal precoding method is the method that allocates power when the direction of precoding vector is given. MRT, ZF and SLNR are well-known precoding method. In this subsection, we illustrate the directions of MRT precoding, SLNR precoding and ZF precoding and utilize convex optimization method to solve problem (7) when the direction of precoding is provided.

1) The Direction Of Suboptimal Precoding: Different precoding methods have different intention. The MRT precoding maximizes the received SNR, the ZF precoding aims at eliminating interference between MTs and the SLNR precoding takes the noise contribution and the interference between MTs into account when choosing $\mathbf{t}_{k}$. The direction of MRT precoding and the direction of channel vector is the same and the performance of MRT precoding is the best if there is no interference. $\tilde{\mathbf{t}}_{k}$, the direction of $\mathbf{t}_{k}$, can be expressed as

$$
\tilde{\mathbf{t}}_{k}^{\mathrm{MRT}}=\frac{\mathbf{h}_{k}}{\left\|\mathbf{h}_{k}\right\|}
$$

In multiuser GDAS, the performance of MRT is visibly deteriorated because of interference from other MTs. ZF precoding method [7] focuses on completely canceling the interference between MTs. The direction of ZF precoding is same as the generalized inverse of channel. The generalized inverse matrix of channel matrix $\mathbf{H}$ is written as $\mathbf{H}^{\dagger}$. Letting $\mathbf{h}_{k}^{\dagger}$ denoting the $k$-th column of $\mathbf{H}^{\dagger}$, the direction of ZF precoding can be expressed as

$$
\tilde{\mathbf{t}}_{k}^{\mathrm{ZF}}=\frac{\mathbf{h}_{k}^{\dagger}}{\left\|\mathbf{h}_{k}^{\dagger}\right\|}
$$

ZF precoding method has not taken noise contributions into account when solving $\tilde{\mathbf{t}}^{\mathrm{ZF}}$. [8] introduces the SLNR precoding in term of so-called signal to leakage noise ratio defined as

$$
\operatorname{SLNR}_{k}=\frac{\left|\mathbf{h}_{k}^{\mathrm{H}} \mathbf{t}_{k}\right|^{2}}{\left.\sum_{i=1, i \neq k} \mathbf{h}_{k}^{\mathrm{H}} \mathbf{t}_{k}\right|^{2}+\sigma_{k}^{2}}
$$

Using the concept of leakage, a new optimization problem is formulated. SLNR precoding deals with the total interference power that user $\mathrm{k}$ causes on all other users. $\tilde{\mathbf{t}}_{k}$, the direction of $\mathbf{t}_{k}$, is given by

$\tilde{\mathbf{t}}_{k}^{\mathrm{SLNR}} \propto \max . \quad$ eigenvector $\left(\left(\mathbf{H}_{k}^{\mathrm{H}} \mathbf{H}_{k}+\sigma_{k}^{2}\right)^{-1} \mathbf{h}_{k}^{\mathrm{H}} \mathbf{h}_{k}\right)$

in terms of the eigenvector corresponding to the largest eigenvalue of matrix $\left(\mathbf{H}_{k}^{\mathrm{H}} \mathbf{H}_{k}+\sigma_{k}^{2}\right)^{-1} \mathbf{h}_{k}^{\mathrm{H}} \mathbf{h}_{k}$. Where, $\mathbf{H}_{k}$ is defined as $\mathbf{H}_{k}=\left[\mathbf{h}_{1} \ldots \mathbf{h}_{k-1} \mathbf{h}_{k+1} \ldots \mathbf{h}_{K}\right]^{\mathrm{T}}$.

2) Power Allocation: The precoding vector can be written as

$$
\mathbf{t}_{k}=\sqrt{p_{k}} \tilde{\mathbf{t}}_{k}
$$

Reformulating problem (7), the new expression can be got

$$
\min _{\mathbf{t}_{1}, \ldots, \mathbf{t}_{K}} \sum_{k=1}^{K} p_{k}
$$

subject to $\frac{1}{\gamma_{0}} p_{k}\left|\mathbf{h}_{k}^{\mathrm{H}} \mathbf{t}_{k}\right|^{2} \geq \sum_{i \neq k}\left|\mathbf{h}_{k}^{\mathrm{H}} \mathbf{t}_{i}\right|^{2}+\sigma_{k}^{2}, k=1, \ldots, K$

The problem (12) is a convex problem and the KKT conditions are used to solve it. The Lagrangian associated with problem (12) is given by 


$$
\mathrm{L}\left(p_{1}, \ldots, p_{K} ; \lambda_{1}, \ldots, \lambda_{K}\right)=\sum_{k=1}^{K} p_{k}+\sum_{k=1}^{K} \lambda_{k}\left(\sum_{i \neq k} p_{i}\left|\mathbf{h}_{k}^{\mathrm{H}} \tilde{\mathbf{t}}_{i}\right|^{2}+\sigma_{k}^{2}-\frac{1}{\gamma_{0}} p_{k}\left|\mathbf{h}_{k}^{\mathrm{H}} \tilde{\mathbf{t}}_{k}\right|^{2}\right)
$$

where $\lambda_{k} \geq 0, k=1, \ldots, K$ are the Lagrange dual variables. Its primal and dual variables are optimal if and only if the following the KKT conditions are satisfied

$$
\begin{aligned}
& \partial \mathrm{L} / \partial p_{k}=\mathbf{0} \Rightarrow 1+\sum_{i \neq k} \lambda_{i}\left|\mathbf{h}_{i} \tilde{\mathbf{t}}_{k}\right|^{2}-\frac{\lambda_{k}}{\gamma_{0}}\left|\mathbf{h}_{k} \mathbb{\mathbf { t }}_{k}\right|^{2}=0 \\
& \lambda_{k}\left(\sum_{i \neq k} p_{i}\left|\mathbf{h}_{k}^{\mathrm{H}} \tilde{\mathbf{t}}_{i}\right|^{2}+\sigma_{k}^{2}-\frac{1}{\gamma_{0}} p_{k}\left|\mathbf{h}_{k}^{\mathrm{H}} \tilde{\mathbf{t}}_{k}\right|^{2}\right)=0 \\
& \sum_{i \neq k} p_{i}\left|\mathbf{h}_{k}^{\mathrm{H}} \tilde{\mathbf{t}}_{i}\right|^{2}+\sigma_{k}^{2}-\frac{1}{\gamma_{0}} p_{k}\left|\mathbf{h}_{k}^{\mathrm{H}} \tilde{\mathbf{t}}_{k}\right|^{2} \leq 0 \\
& \lambda_{k} \geq 0 \quad k=1, \ldots, K
\end{aligned}
$$

If $\lambda_{k}=0$, the KKT conditions are not satisfied. If $\lambda_{k}>0$, the problem is strictly feasible and the optimal dual variables can be given by

$$
\left[\begin{array}{c}
\lambda_{1} \\
\vdots \\
\lambda_{K}
\end{array}\right]=\mathbf{W}^{-1}\left[\begin{array}{c}
1 \\
\vdots \\
1
\end{array}\right] \quad[\mathbf{W}]_{i j}= \begin{cases}\frac{1}{\gamma_{0}}\left|\mathbf{h}_{i}^{\mathrm{H}} \tilde{\mathbf{t}}_{i}\right|^{2} & i=j \\
-\left|\mathbf{h}_{j}^{\mathrm{H}} \tilde{\mathbf{t}}_{i}\right|^{2} & i \neq j\end{cases}
$$

Moreover, the optimal variables can be expressed as

$$
\left[\begin{array}{c}
p_{1} \\
\vdots \\
p_{K}
\end{array}\right]=\left(\mathbf{W}^{\mathrm{T}}\right)^{-1}\left[\begin{array}{c}
\sigma_{1}^{2} \\
\vdots \\
\sigma_{K}^{2}
\end{array}\right]
$$

\section{Simulation And Analysis}

\begin{tabular}{|c|c|}
\hline Parameters & Value \\
\hline$D$ & $500 \mathrm{~m}$ \\
\hline$d_{0}$ & $35 \mathrm{~m}$ \\
\hline$\alpha$ & 3.5 \\
\hline$f_{c}$ & $900 \mathrm{MHz}$ \\
\hline$\sigma_{\mathrm{dB}}$ & $6 \mathrm{~dB}$ \\
\hline$N_{0}$ & $-174 \mathrm{dBm} / \mathrm{Hz}$ \\
\hline$N$ & 7 \\
\hline$L$ & 1 \\
\hline$K$ & 4 \\
\hline
\end{tabular}

TABLE I. Simulation Parameters

The simulation scenario shown in Fig.1 is considered and parameters are showed in the TABLE I. We define outage as the event that the transmission power, as the solution to (5) or (16), is larger than a threshold $P_{\text {max }}$, that is $\operatorname{Pr}\left(P_{\mathrm{tx}}>P_{\text {max }}\right)$ where $P_{\mathrm{tx}}=\sum_{\mathrm{k}}\left\|\mathbf{t}_{k}\right\|^{2}$ in term of the optimal precoding problem (5) or $P_{\mathrm{tx}}=\sum_{\mathrm{k}} p_{k}$ in term of the suboptimal precoding problem (16). Outage analysis quantifies the level of performance that is guaranteed with a certain level of reliability. Accordingly, we define $q \%$-outage transmission power $P_{q \% \text {-outage }}$ as the power threshold that can be expressed as

$$
\operatorname{Pr}\left(P_{\mathrm{tx}}>P_{q \% \text {-outage }}\right)=q \%
$$

In the following discussion, the outage probability of all linear precoding methods are analyzed and the cumulative distribution functions (CDF) of transmission power is also illustrated. we also compared the outage probability and outage transmission power in different systems.

Fig. 2 presents the outage probability of different linear precoding methods when $P_{\text {max }}$ is $34 \mathrm{dBW}$. As we can see in this figure, the outage probability of ZF precoding is higher than other precoding methods when SINR is less $2 \mathrm{~dB}$. ZF precoding eliminates the interference between users, but also 
enhances the noise. This explains why ZF precoding needs more transmission power. With the increasing of $\gamma_{0}$, the outage probability of MRT precoding and SLNR precoding are higher than the outage probability of ZF precoding. This is because MRT precoding and SLNR precoding are not fair enough to meet all MTs. Compared with other precoding methods, the outage probability of optimal precoding is minimal. Fig. 3 shows the cumulative distribution function (CDF) of transmission power when $\gamma_{0}=0 \mathrm{~dB}$. Along with the increasing of transmission power, the CDF of optimal precoding and ZF precoding is gradually closing to 1 but the CDF of MRT precoding and SLNR precoding gradually approach 0.9. This phenomenon show that MRT precoding and SLNR precoding can not satisfy SINR constraints. In other words, the problem (12) probably has no solutions when using MRT precoding or SLNR precoding. This illustrates the unfairness of SLNR precoding and MRT precoding. From Fig. 3, we also can get the conclusion that the optimal precoding needs less transmission power for the same $\gamma_{0}$ compared with other precoding methods. From Fig. 2 and Fig. 3, the performance of optimal precoding method is the best compared with other precoding methods.

As shown in Fig. 4, when using the same precoding, the outage probability of CAS is higher than that of DAS. With $\gamma_{0}$ growing, the gap between the CAS and the DAS is more. The Fig. 5 depicts the outage probability when the MTs located in cell edge. Here, the cell edge is defined as the region that is $2 / 3 D$ from the center of cell. From Fig. 5, with $\gamma_{0}$ growing, the outage probability of CAS is closed to 1 and the outage probability of DAS is approached 0.8. This mean that, compared with CAS, in cell edge, the DAS has tremendous advantage in improving the SINR. Through Fig. 4 and Fig. 5, we can get the conclusion that the DAS can improve coverage compared with the CAS and the DAS is easier to satisfy high SINR constraints in cell edge.

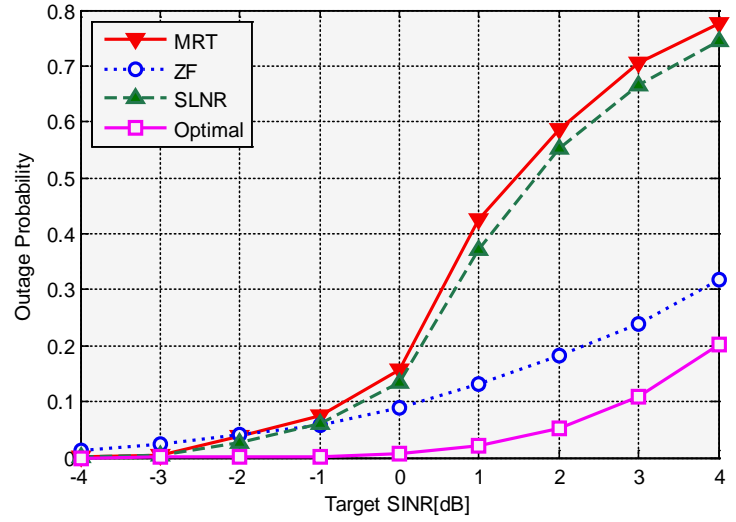

Fig.2. The outage probability in DAS

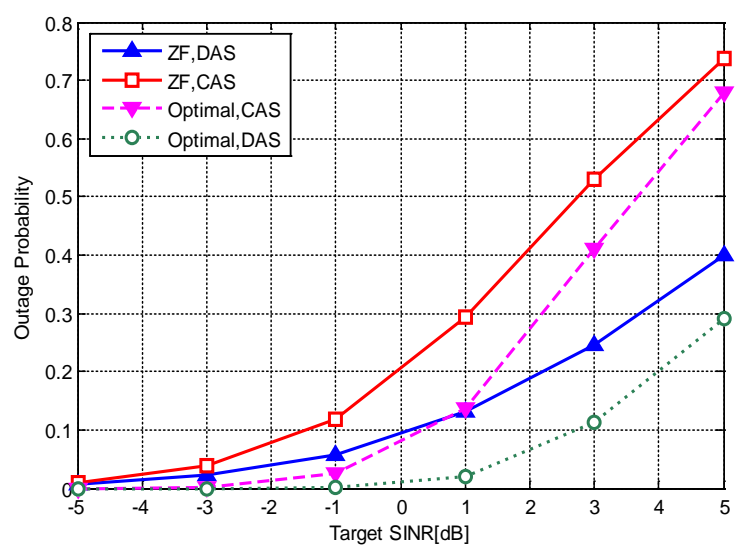

Fig.4. The outage probability

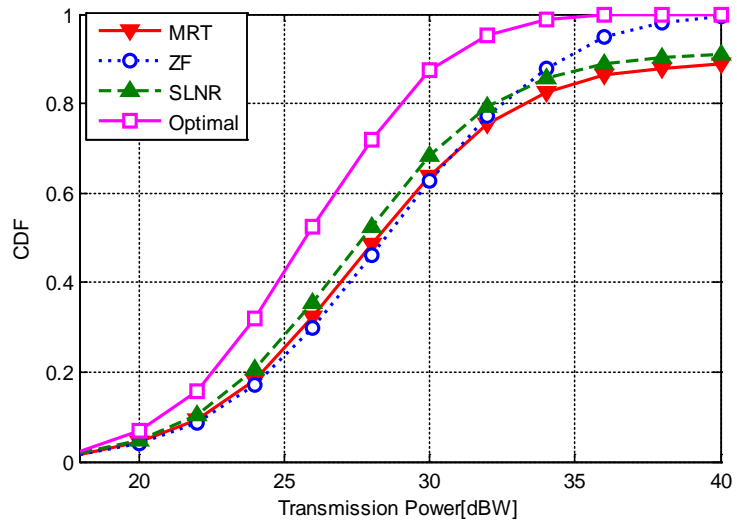

Fig.3. The CDF in DAS

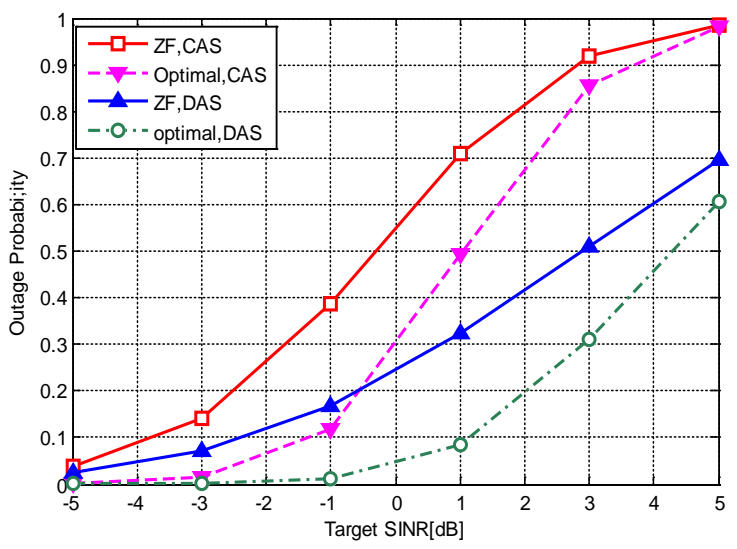

Fig.5. The outage probability in edge 


\section{Conclusion}

This paper introduced the system model of distributed antenna system and the linear precoding. we solved the problem that minimize the transmission power with SINR constraints in DAS and design the optimal precoding. Furthermore, we introduced the power allocation and designed the suboptimal precoding methods. In simulation, we compared the optimal precoding with suboptimal precoding and found the advantage of optimal precoding in reducing transmission power. Furthermore, we compared the DAS with the CAS using different precoding methods and got the conclusion that the DAS has advantages in reducing transmission power and improving the coverage.

\section{Acknowledgement}

This work was supported by National Natural Science Foundation of China under grants 61571120 and 61501113, and by Natural Science Foundation of Jiangsu Province under grants BK20151415 and BK20150630.

\section{References}

[1] You X, Wang D, Zhu P, et al. Cell edge performance of cellular mobile systems[J]. Selected Areas in Communications, IEEE Journal on, 2011, 29(6): 1139-1150.

[2] Saleh A A M, Roman R S. Distributed antennas for indoor radio communications[J]. Communications, IEEE Transactions on, 1987, 35(12): 1245-1251.

[3] Roh W, Paulraj A. MIMO channel capacity for the distributed antenna[C] VTC 2002-Fall. 2002 IEEE 56th. IEEE, 2002, 2: 706-709.

[4] Dai L, Zhou S, Yao Y. Capacity analysis in CDMA distributed antenna systems[J]. Wireless Communications, IEEE Transactions on, 2005, 4(6): 2613-2620.

[5] Choi W, Andrews J G. Downlink performance and capacity of distributed antenna systems in a multicell environment[J]. Wireless Communications, IEEE Transactions on, 2007, 6(1): 69-73.

[6] Lo T K Y. Maximum ratio transmission[C] 1999. ICC'99. 1999 IEEE International Conference on. IEEE, 1999, 2: 1310-1314.

[7] Wiesel A, Eldar Y C, Shamai S. Zero-forcing precoding and generalized inverses[J]. Signal Processing, IEEE Transactions on, 2008, 56(9): 4409-4418.

[8] Sadek M, Tarighat A, Sayed A H. A leakage-based precoding scheme for downlink multi-user MIMO channels[J]. Wireless Communications, IEEE Transactions on, 2007, 6(5): 1711-1721.

[9] Björnson E, Bengtsson M, Ottersten B. Optimal multiuser transmit beamforming: A difficult problem with a simple solution structure[J]. arXiv preprint arXiv:1404.0408, 2014.

[10] Lobo M S, Vandenberghe L, Boyd S, et al. Applications of second-order cone programming[J]. Linear algebra and its applications, 1998, 284(1): 193-228.

[11] Sturm J F. Using SeDuMi 1.02, a MATLAB toolbox for optimization over symmetric cones[J]. Optimization methods and software, 1999, 11(1-4): 625-653.

[12] Khachan A M, Tenenbaum A J, Adve R S. Linear processing for the downlink in multiuser MIMO systems with multiple data streams[C] 2006. ICC'06. IEEE International Conference on. IEEE, 2006, 9: 4113-4118.

[13] Schubert M, Boche H. Solution of the multiuser downlink beamforming problem with individual SINR constraints[J]. Vehicular Technology, IEEE Transactions on, 2004, 53(1): 18-28. 